\title{
Two-Dimensional Speckle Tracking Echocardiography Detects Subclinical Left Ventricular Systolic Dysfunction among Adult Survivors of Childhood, Adolescent, and Young Adult Cancer
}

\author{
Anthony F. Yu, ${ }^{1,2}$ Jayant Raikhelkar, ${ }^{1}$ Emily C. Zabor, ${ }^{3}$ Emily S. Tonorezos, ${ }^{1,2}$ \\ Chaya S. Moskowitz, ${ }^{3}$ Roberto Adsuar, ${ }^{1}$ Elton Mara, ${ }^{1}$ Kevin Huie, ${ }^{1}$ Kevin C. Oeffinger, ${ }^{1,2}$ \\ Richard M. Steingart, ${ }^{1,2}$ and Jennifer E. Liu ${ }^{1,2}$ \\ ${ }^{1}$ Department of Medicine, Memorial Sloan Kettering Cancer Center, New York, NY 10065, USA \\ ${ }^{2}$ Weill Cornell Medical Center, New York, NY 10065, USA \\ ${ }^{3}$ Department of Epidemiology and Biostatistics, Memorial Sloan Kettering Cancer Center, New York, NY 10065, USA
}

Correspondence should be addressed to Anthony F. Yu; yua3@mskcc.org

Received 15 October 2015; Accepted 11 January 2016

Academic Editor: Giovanni Di Salvo

Copyright (C) 2016 Anthony F. Yu et al. This is an open access article distributed under the Creative Commons Attribution License, which permits unrestricted use, distribution, and reproduction in any medium, provided the original work is properly cited.

\begin{abstract}
Two-dimensional speckle tracking echocardiography (2DSTE) provides a sensitive measure of left ventricular (LV) systolic function and may aid in the diagnosis of cardiotoxicity. 2DSTE was performed in a cross-sectional study of 134 patients (mean age: $31.4 \pm$ 8.8 years; $55 \%$ male; mean time since diagnosis: $15.4 \pm 9.4$ years) previously treated with anthracyclines (mean cumulative dose: $\left.320 \pm 124 \mathrm{mg} / \mathrm{m}^{2}\right)$, with $(n=52)$ or without $(n=82)$ mediastinal radiotherapy. The prevalence of LV systolic dysfunction, defined as fractional shortening $<27 \%$, LV ejection fraction (LVEF) $<55 \%$, and global longitudinal strain (GLS) $\leq 16 \%$, was $5.2 \%, 6.0 \%$, and $23.1 \%$, respectively. Abnormal GLS was observed in $24(18 \%)$ patients despite a normal LVEF. Indices of LV systolic function were similar regardless of anthracycline dose. However, GLS was worse (18.0 versus 19.0, $p=0.003)$ and prevalence of abnormal GLS was higher $(36.5 \%$ versus $14.6 \%, p=0.004)$ in patients treated with mediastinal radiotherapy. Mediastinal radiotherapy was associated with reduced GLS ( $p=0.040)$ after adjusting for sex, age, and cumulative anthracycline dose. In adult survivors of childhood, adolescent, and young adult cancer, 2DSTE frequently detects LV systolic dysfunction despite a normal LVEF and may be useful for the long-term cardiac surveillance of adult cancer survivors.
\end{abstract}

\section{Introduction}

Strategies in cancer diagnostics and therapeutics have improved dramatically over the last several decades, leading to a growing number of adult survivors of childhood, adolescent, and young adult cancer. The 5-year survival rate for all cancers diagnosed before age of 45 exceeds $80 \%$ [1]. As of 2008 , there were 619,000 survivors of cancer under the age of 40 years [2]. Despite this progress, the long-term noncancer mortality rate in this population remains substantially higher than age- and gender-matched controls [3], with cardiovascular disease as one of the leading causes of death behind disease recurrence and secondary malignancy $[4,5]$. Survivors of childhood (age of 0-14 years) and adolescent (age of 15-19 years) cancer are 7 times more likely to die from cardiacrelated events compared to age- and sex- specific rates in the US population [3,6] and 15.1 times more likely than their siblings to develop heart failure [7]. Less is known about the cardiac outcomes in survivors of young adult (age of 20-39 years) cancer. 
Late cardiac effects due to anthracycline chemotherapy and mediastinal radiotherapy in adult cancer survivors are well recognized [8], and subclinical changes in left ventricular (LV) structure and/or function may be seen in more than half of survivors exposed to cardiotoxic therapy [911]. Anthracycline chemotherapy can lead to dilated cardiomyopathy and heart failure [12]. Mediastinal radiotherapy is associated with heart failure, premature coronary artery disease, pericardial injury, arrhythmias, and valvular abnormalities $[13,14]$. Determination of left ventricular ejection fraction (LVEF) by two-dimensional (2D) transthoracic echocardiography is the primary modality used to screen for LV systolic dysfunction. The Children's Oncology Group (COG) and the National Comprehensive Cancer Network (NCCN) recommend screening echocardiography for all survivors of childhood, adolescent, and young adult cancer, with a frequency based on age at first cardiotoxic treatment, exposure to cardiac-directed radiotherapy, and cumulative anthracycline dose $[15,16]$. However, due to the inherent interobserver and intraobserver variability of LVEF assessment by echocardiography, a change in LVEF of $9-11 \%$ is the minimum that can be recognized with $95 \%$ confidence [17]. In addition, a decline in LVEF is considered to be a late finding of cardiotoxicity $[18,19]$.

Two-dimensional speckle tracking echocardiography (2DSTE) is a novel method that has potential advantages over LVEF or FS measurement in the early detection of LV systolic dysfunction among both children [20,21] and adults $[22,23]$ treated with cardiotoxic therapies and is recommended by the American Society of Echocardiography for the evaluation of patients during and after cancer therapy [24]. However, less is known regarding the use of 2DSTE for the detection of LV systolic dysfunction among adult survivors of childhood, adolescent, and young adult cancer. We performed a single institution cross-sectional study to investigate the use of 2DSTE to detect LV systolic dysfunction among adult survivors of childhood, adolescent, and young adult cancer treated with prior anthracycline chemotherapy, with or without mediastinal radiotherapy.

\section{Methods}

2.1. Study Population. A total of 134 consecutively referred adult survivors of childhood, adolescent, and young adult cancer followed up in the Adult Long-Term Follow-Up Program at Memorial Sloan Kettering Cancer Center, a program for high-risk cancer survivors, were included in this study. Screening echocardiograms were performed between July 1, 2010, and December 31, 2012. As per COG and NCCN recommendations, screening echocardiograms were performed annually or biennially $[15,16]$. All patients were previously treated with anthracycline chemotherapy, with or without mediastinal radiotherapy, and did not have any known history of symptomatic heart failure. The following data was extracted retrospectively from medical records for each patient: age at diagnosis, cancer diagnosis, date of diagnosis, cardiac risk factors (hypertension, diabetes mellitus, and dyslipidemia), cumulative anthracycline dose, total mediastinal radiotherapy dose, and use of cardiac medications (including aspirin, beta blockers, angiotensin converting enzyme (ACE) inhibitors, angiotensin receptor blockers, or statins). As previously described [15], the cumulative isotoxic anthracycline dose was calculated as the sum of the following: doxorubicin $+($ daunorubicin $\times 0.833)+$ $($ epirubicin $\times 0.57)+($ idarubicin $\times 5)+($ mitoxantrone $\times 4$ ). Mediastinal radiotherapy was defined as any form of radiotherapy in which the myocardium was within the prespecified radiation field.

2.2. LV Function by 2D Echocardiography. Conventional 2D and Doppler echocardiography was performed using a commercially available standard ultrasound scanner (Vivid E9, General Electric Medical Systems, Milwaukee, WI), according to the standardized American Society Echocardiography (ASE) protocol [25]. LVEF was calculated using the modified Simpson's method. Fractional shortening (FS) was calculated using the standard equation. Abnormal LV systolic function was defined as LVEF $<55 \%$ or FS $<27 \%$. Mitral inflow velocity pattern was recorded from the apical 4 -chamber view with the pulsed-wave Doppler sample volume positioned at the tips of the leaflets during diastole. Peak early ( $E$-wave) and late $(A$-wave) diastolic filling velocities were measured and their ratio (mitral $E / A$ ) was calculated. Doppler tissue imaging of the mitral annulus was performed with measurement of the early $\left(e^{\prime}\right)$ diastolic velocity at the septal annulus. Diastolic function assessment was based on mitral $E / A$ ratio and tissue Doppler $\left(e^{\prime}\right)$ velocity only.

\subsection{D Speckle Tracking Echocardiography. Three apical} views were used to measure peak systolic global longitudinal strain (GLS) and strain rate using dedicated automated software (EchoPAC 12.0, GE Healthcare, Milwaukee, WI). Three points were manually placed at the endocardial border (one in the apex and two at the mitral valve annulus) in each of the apical views, allowing the software to automatically track myocardial movement throughout the cardiac cycle. After careful inspection, manual correction was performed if the automatic detection was suboptimal. Each view was divided into 6 segments, for a total of 18 segments representing the entire left ventricle. In the case of unsatisfactory tracking due to inadequate image quality, that segment was eliminated from analysis. The timing of aortic valve closure in the apical 3-chamber view was used to define end-systole. Longitudinal strain and strain rate curves were generated for each segment. Strain data were expressed in absolute values $(|\%|)$. Global radial (GRS) and circumferential (GCS) strain were calculated by averaging the peak systolic strain values in all 6 segments of the parasternal short-axis view at the papillary muscle. Abnormal LV systolic function was defined as GLS $\leq|16| \%$. This is a conservative estimate of the lower limit of normal that was derived from studies performed in several healthy cohorts using the mean GLS minus two standard deviations [23, 26, 27].

Interobserver variability was assessed by comparing the original GLS, GRS, and GCS calculation with that calculated by a blinded second observer in 20 randomly selected patients. Intraobserver variability was calculated by repeated 
measurements in 20 patients by the primary reviewer 3 weeks after the initial measurement.

2.4. Statistical Analysis. Continuous measures were summarized as median and interquartile range (IQR) whereas categorical measures were summarized as frequency and percent. Patients were stratified based on cumulative anthracycline dose $\left(<250,250-399\right.$, and $\left.\geq 400 \mathrm{mg} / \mathrm{m}^{2}\right)$ and by mediastinal radiotherapy (yes versus no) for the comparison of the $2 \mathrm{D}$ echocardiographic and strain indices, and by-group comparisons were tested using Fisher's exact test when categorical and Kruskal-Wallis test when continuous. The prevalence of abnormal GLS and abnormal FS and LVEF was compared using an exact McNemar's test. Dependent variables considered in multivariable linear regression analysis included FS, LVEF, LV mass/BSA, GLS, mitral $E / A$ ratio, and septal $e^{\prime}$ velocity. GLS $\leq|16| \%$ was considered the dependent variable in a multivariable logistic regression model. There were too few events to consider FS $<27 \%$ or EF $<55 \%$ as outcomes in multivariable analysis. Each multivariable regression model incorporated sex, age at echocardiogram, mediastinal radiotherapy (yes or no), and cumulative anthracycline dose (continuous) as independent variables. Interobserver and intraobserver variability were assessed using the intraclass correlation coefficient (ICC). Bland-Altman plots were constructed by plotting the average of the two readings on the $x$-axis versus the difference between the two readings on the $y$-axis. The mean and standard deviation of the differences were calculated. Because of the small sample size, the $t$-distribution was used as the reference distribution to calculate the $95 \%$ limits of agreement. A $p$ value of less than 0.05 was considered statistically significant. All statistical analyses were performed using either SAS software version 9.2 (SAS Institute, Cary, NC) or R software version 2.13.1 (R Core Development Team, Vienna, Austria).

\section{Results}

3.1. Patient Characteristics. A total of 134 survivors of childhood, adolescent, and young adult cancer were included in this study. Demographic and treatment characteristics are provided in Table 1. The median age at echocardiographic follow-up was 31 years (range, 18 to 62 years), and the median interval since diagnosis was 15 years (range, 2 to 39 years). Sarcoma $(n=54)$, Hodgkin lymphoma $(n=$ 29 ), and acute leukemia $(n=31)$ were the most common diagnoses. All patients were treated with anthracycline chemotherapy, and the median cumulative anthracycline dose was $300 \mathrm{mg} / \mathrm{m}^{2}$ (range, 27 to $660 \mathrm{mg} / \mathrm{m}^{2}$ ). Less than half of patients $(39 \%)$ received mediastinal radiotherapy as part of their cancer treatment, with a median dose of $35 \mathrm{~Gy}$ (range, 2 to $56 \mathrm{~Gy}$ ). The overall prevalence of cardiac risk factors such as hypertension and diabetes was low $(9.0 \%$ and $5.2 \%$, resp.). Patients who received mediastinal radiotherapy as part of their cancer treatment regimen $(n=52)$ had a lower cumulative anthracycline dose exposure (median dose, 279 versus $375 \mathrm{mg} / \mathrm{m}^{2}, p<0.001$ ) compared to those who were treated with anthracycline chemotherapy alone.
3.2. LV Structure and Function. Overall, the mean FS, LVEF, and GLS were $33.3 \%$ (range, $23.8 \%$ to $44.1 \%$ ), $61.1 \%$ (range, $46.9 \%$ to $75.6 \%$ ), and $18.0 \%$ (range, $12 \%$ to $26 \%$ ). LV systolic dysfunction was detected by FS $<27 \%$ in $5.2 \%$ and LVEF $<55 \%$ in $6.0 \%$. However, the prevalence of abnormal GLS $\leq|16| \%$ was $23.1 \%$, significantly higher than abnormal FS $(p<0.001)$ or LVEF $(p<0.001)$ (Figure 1$)$. In the 31 patients with abnormal GLS, only 7 had an abnormal LVEF. There was no significant association between echocardiographic parameters of LV systolic or diastolic function (by $2 \mathrm{D}$ echocardiography or 2DSTE) and cumulative anthracycline dose (data not shown).

Echocardiographic parameters of LV systolic function, stratified by mediastinal radiotherapy exposure, are shown in Table 2. GLS was worse in the mediastinal radiotherapy group as compared to the nonradiotherapy group (18\% versus $19 \%$, $p=0.003)$ and the prevalence of patients with GLS $\leq|16| \%$ was more than two times greater in the radiotherapy group (36.5\% versus $14.6 \%, p=0.004)$. However, there was no difference in FS $(33.3 \%$ versus $33.1 \%, p=0.686)$ or LVEF (60.7 versus $61.2 \%, p=0.301)$ between the groups with and without mediastinal radiotherapy. Although GCS for the whole study group was significantly lower than the normative reference value (mean 23.3\%; 95\% confidence interval [CI] $22.1 \%$ to $24.6 \%$ ) based on a recent meta-analysis of normal ranges [28], there was no difference in GCS $(16.1 \%$ versus $17.6 \%, p=0.203)$ or GRS $(42.1 \%$ versus $42.0 \%, p=$ 0.843 ) between the groups with and without mediastinal radiotherapy.

In multivariable linear regression analysis, mediastinal radiotherapy was associated with reduced GLS (beta $=0.923$, standard error $=0.444, p=0.040$ ) after adjusting for sex, age at echocardiogram, and cumulative anthracycline dose. Mediastinal radiotherapy was also associated with decreased indices of diastolic function, including lower transmitral $E / A$ ratio (beta $=-0.250$, standard error $=0.087 ; p=$ 0.005 ) and medial septal tissue Doppler $e^{\prime}$ velocity (beta $=$ -1.221 , standard error $=0.404 ; p=0.003$ ). There was a trend towards increased LV mass index with higher doses of anthracycline chemotherapy (beta $=0.702$, standard error $=0.404 ; p=0.085)$ and decreased LV mass index with mediastinal radiotherapy (beta $=-3.459$, standard error $=$ 2.085; $p=0.099$ ), but neither association reached statistical significance.

3.3. Intraobserver and Interobserver Variability. For GLS, the ICC for interobserver agreement was 0.837 (95\% CI 0.639 to 0.932 ) and the ICC for intraobserver agreement was 0.822 (95\% CI 0.610 to 0.925 ), reflecting substantial agreement for measurement of GLS. The Bland-Altman plots indicate that, for interobserver agreement, we expect $95 \%$ of measurements from two different readers to differ by between $-1.86 \%$ and $2.28 \%$ whereas, for intraobserver agreement, we expect $95 \%$ of measurements taken by the same reader at two times to differ by between $-0.84 \%$ and $2.35 \%$ (Figure 2 ). For GCS, the ICC for interobserver agreement was 0.74 (95\% CI 0.45 to 0.89 ) and the ICC for intraobserver agreement was 0.93 (95\% CI 0.82 to 0.97 ). For GRS, the ICC for interobserver 
TABLE 1: Demographics and treatment characteristics $(n=134)^{*}$.

\begin{tabular}{|c|c|c|c|c|}
\hline Characteristic & No. & $\%$ & Mean & SD \\
\hline Current age, years & & & 31.4 & 8.8 \\
\hline Median & & & & \\
\hline Range & & & & \\
\hline Age at diagnosis, years & & & 16.0 & 9.0 \\
\hline Median & & & & \\
\hline Range & & & & \\
\hline Interval since diagnosis, years & & & 15.4 & 9.4 \\
\hline Median & & & & \\
\hline Range & & & & \\
\hline \multicolumn{5}{|l|}{ Sex } \\
\hline Male & 73 & 54.5 & & \\
\hline Female & 61 & 45.5 & & \\
\hline \multicolumn{5}{|l|}{ Race } \\
\hline Non-Hispanic white & 114 & 85.1 & & \\
\hline Black & 9 & 6.7 & & \\
\hline Other & 11 & 8.2 & & \\
\hline \multicolumn{5}{|l|}{ Diagnosis } \\
\hline Sarcoma & 54 & 40.2 & & \\
\hline Hodgkin lymphoma & 29 & 21.6 & & \\
\hline Acute lymphoblastic leukemia & 17 & 12.7 & & \\
\hline Acute myeloid leukemia & 14 & 10.4 & & \\
\hline Non-Hodgkin lymphoma & 9 & 6.7 & & \\
\hline Other $^{\dagger}$ & 11 & 8.2 & & \\
\hline Anthracycline cumulative dose exposure & & & 320 & 124 \\
\hline Median & & & & \\
\hline Range & & & & \\
\hline$>350$ & 57 & 42.5 & & \\
\hline $150-350$ & 58 & 43.3 & & \\
\hline $1-150$ & 19 & 14.2 & & \\
\hline \multicolumn{5}{|l|}{ Mediastinal RT dose, Gy } \\
\hline None & 82 & 61.2 & & \\
\hline $1-30$ & 38 & 28.4 & & \\
\hline$>30$ & 14 & 10.4 & & \\
\hline \multicolumn{5}{|l|}{ Cardiovascular risk factors } \\
\hline Hypertension & 12 & 9.0 & & \\
\hline Diabetes & 7 & 5.2 & & \\
\hline Dyslipidemia & 42 & 31.3 & & \\
\hline Treatment with beta-blockers or ACE-I & 14 & 10.4 & & \\
\hline Body mass index & & & 24.8 & 4.7 \\
\hline Median & & & & \\
\hline Range & & & & \\
\hline Overweight (BMI 25-<30) & 39 & 29.1 & & \\
\hline Obese $(\mathrm{BMI} \geq 30)$ & 17 & 12.7 & & \\
\hline
\end{tabular}

${ }^{*} \mathrm{RT}$, radiotherapy; ACE-I, angiotensin converting enzyme inhibitor; BMI, body mass index; SD, standard deviation.

${ }^{\dagger}$ Other: neuroblastoma $(n=3)$, chronic myeloid leukemia $(n=2)$, teratoma $(n=2)$, ependymoma $(n=1)$, nasopharyngeal carcinoma $(n=1)$, retinoblastoma $(n=1)$, and Wilms' tumor $(n=1)$.

agreement was 0.83 (95\% CI 0.63 to 0.93 ) and the ICC for intraobserver agreement was 0.77 (95\% CI 0.51 to 0.90 ).

\section{Discussion}

The principle finding of this study is that a significant proportion of adult survivors of childhood, adolescent, and young adult cancer have abnormal LV systolic function detected by 2DSTE despite having a normal LVEF. While an abnormal GLS $(\leq|16| \%)$ was observed in nearly one-quarter of study participants, a corresponding reduction in FS of $<27 \%$ or LVEF of $<55 \%$ was observed in only $5.2 \%$ and $6.0 \%$ of participants, respectively. These observations suggest that the prevalence of LV systolic dysfunction among long-term cancer survivors may be significantly underestimated using LVEF alone as compared to GLS by 2DSTE. 
TABLE 2: LV size and function by receipt of mediastinal radiotherapy*.

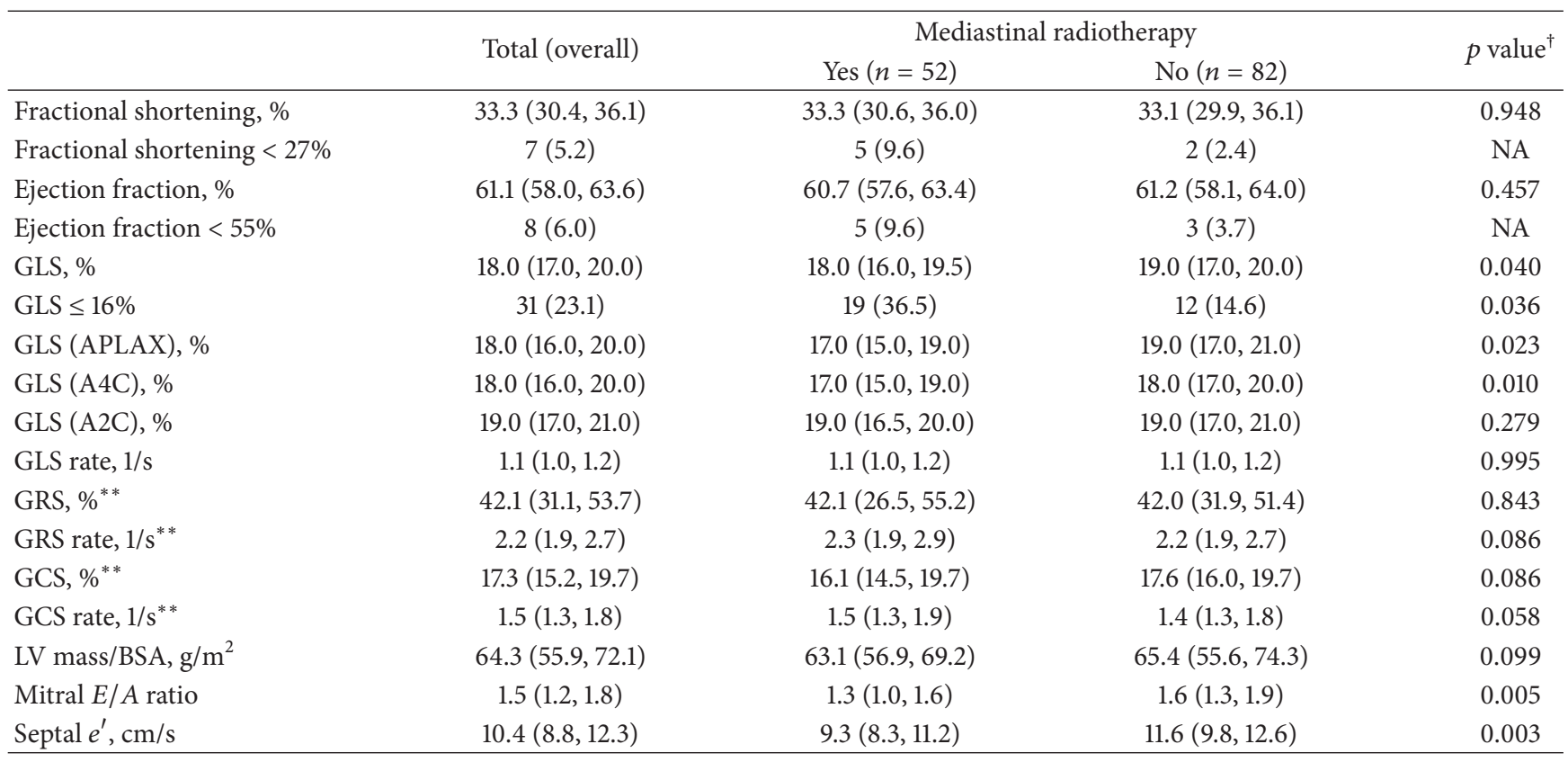

* Numbers are median (interquartile range) for continuous variables and $N(\%)$ for categorical variables. GLS, global longitudinal strain; APLAX, apical long axis; A4C, apical 4-chamber; A2C, apical 2 -hamber; LV, left ventricle; BSA, body surface area. ${ }^{\dagger} p$ value from multivariable linear or logistic regression adjusted for continuous cumulative anthracycline dose, sex, and age at echocardiogram.

*** Based on only $n=130$ patients.

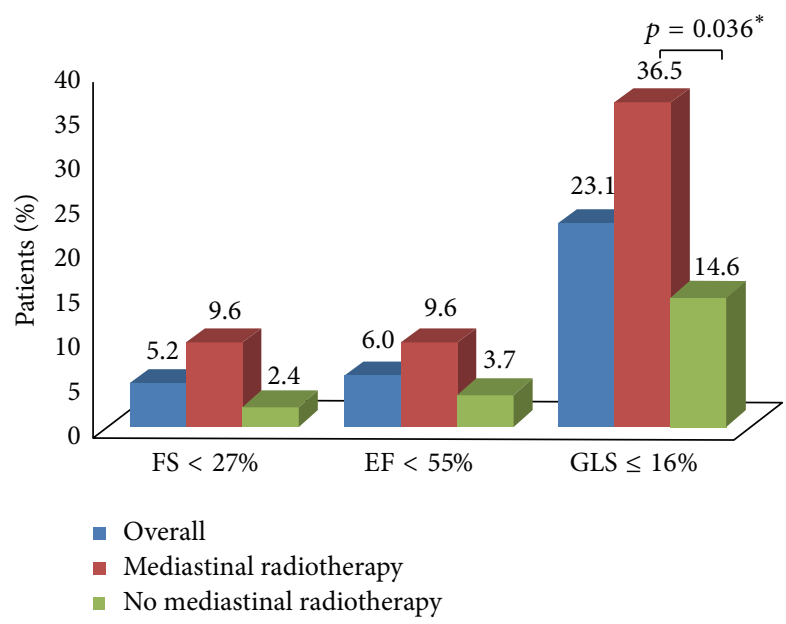

Figure 1: Prevalence of left ventricular systolic dysfunction as measured by left ventricular ejection fraction (LVEF), fractional shortening (FS), and global longitudinal strain (GLS), with or without mediastinal radiotherapy. ${ }^{*} p$ value from multivariable linear or logistic regression adjusted for continuous cumulative anthracycline dose, sex, and age at echocardiogram.

In the current study, there was no association between cumulative anthracycline dose and conventional or strain indices of LV systolic function. However, this may be attributable to the heterogeneity of the study population and the limited sample size. Indices of diastolic function were significantly lower among patients treated with mediastinal radiotherapy (median dose $35 \mathrm{~Gy}$ ). This is consistent with a study by Heidenreich et al. in which a high prevalence of diastolic dysfunction was observed among Hodgkin lymphoma survivors treated with at least $35 \mathrm{~Gy}$ of mediastinal radiotherapy [29]. Interestingly, the presence of diastolic dysfunction was associated with a poorer cardiac event-free survival.

Although LVEF by 2D echocardiography is the current standard method for monitoring LV systolic function in cancer survivors, myocardial strain by 2DSTE has several important advantages as a screening tool for cardiotoxicity. First, myocardial strain can identify subclinical LV systolic dysfunction prior to a decrement of LVEF. Poterucha et al. found that a reduction in GLS preceded a decrease in LVEF among adolescents receiving anthracycline chemotherapy [21]. Similar results were reported in a study of women with HER2-positive breast cancer in which GLS < |19|\% was predictive of subsequent cardiotoxicity and decreased LVEF [30]. In addition to being a more sensitive marker of LV systolic function, GLS has also been shown to be a predictor of all-cause mortality that may be superior to LVEF or wall motion score index [31]. And, consistent with previous reports, the current study shows excellent reproducibility for measures of peak GLS, as represented by interobserver and intraobserver ICCs of $\geq 0.80$.

Findings from several prior studies support the utility of myocardial strain assessment in cancer survivors [3235]. Cheung et al. demonstrated that GLS was reduced among asymptomatic children previously treated for acute lymphoblastic leukemia with anthracycline chemotherapy despite having normal fractional shortening [20]. Tsai et al. also showed that GLS was lower among long-term survivors 


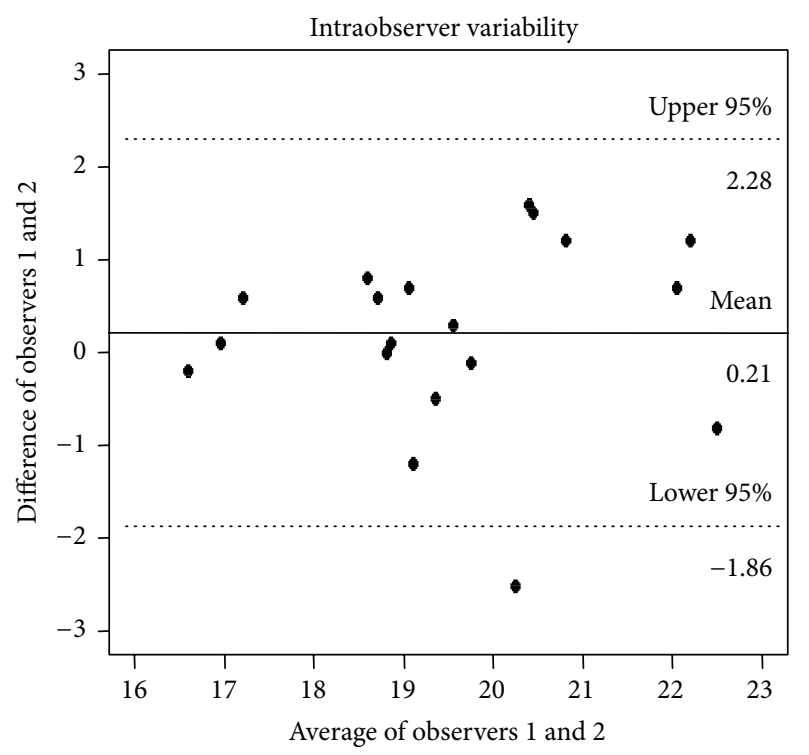

(a)

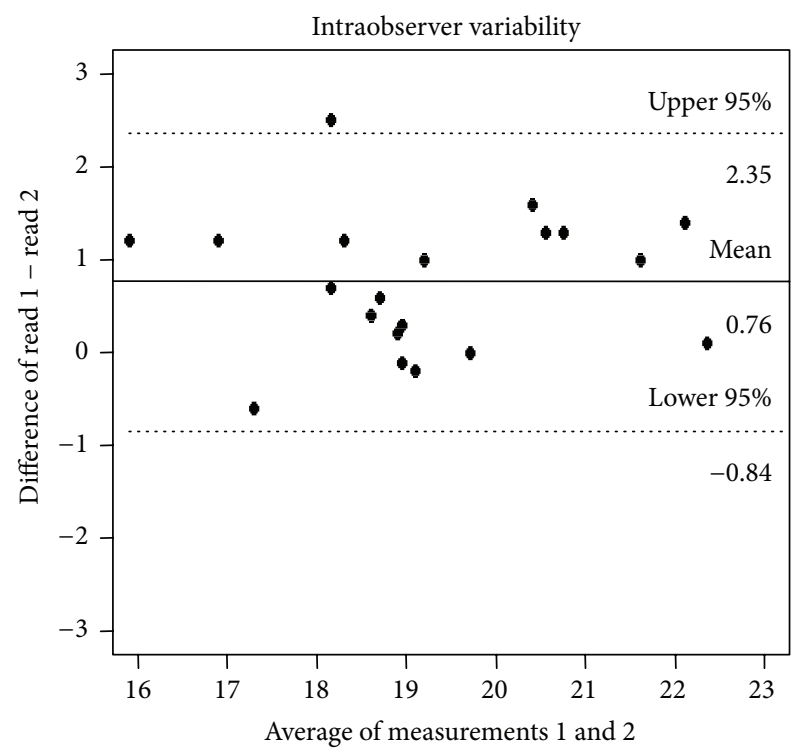

(b)

FIGURE 2: Bland-Altman analysis for interobserver (a) and intraobserver variability (b) for global longitudinal strain measurements in 20 randomly selected patients.

of Hodgkin lymphoma after treatment with anthracycline chemotherapy and mediastinal radiotherapy compared to mediastinal radiotherapy alone [36]. In the current study, GLS was significantly lower and the prevalence of LV systolic dysfunction by 2DSTE was more than two times greater among patients who received both mediastinal radiotherapy and anthracycline treatment compared to those with anthracycline treatment alone. This is consistent with the general finding that longitudinal LV mechanics, which are primarily governed by the subendocardial layer, are the most sensitive and vulnerable to myocardial disease. The circumferential function, which is predominantly governed by the midwall and subepicardial regions, may vary depending on the severity of myocardial involvement. The lack of difference in circumferential strain between the groups with and without radiotherapy may be contributed by the lower reproducibility and accuracy of the circumferential strain measurements.

Less than $10 \%$ of the patients were treated with cardioprotective medications, which may confound the association between mediastinal radiotherapy and GLS. However the results of our analysis were not substantively different when these patients were excluded. While radiation increases the cardiotoxicity of anthracyclines via different mechanisms of injury, it is unclear whether this relationship is additive or synergistic.

Overall, 24 of 134 patients (18\%) in the current study had abnormal GLS despite a normal LVEF. This is consistent with findings from the St. Jude Lifetime Cohort Study, in which abnormal GLS was more prevalent than a reduction in LVEF among adult survivors of childhood cancer and was associated with both chest-directed radiotherapy and anthracycline exposure [37]. Although GLS has been identified as a robust parameter for early detection and prediction of cardiotoxicity during cancer therapy, the prognostic significance of abnormal GLS among survivors after completion of cancer therapy remains unknown [38]. A recent expert consensus statement from the American Society of Echocardiography highlights the utility of 2DSTE to detect early subclinical LV systolic dysfunction related to cancer treatment, which may allow for better cardiac risk stratification and facilitate timely intervention [24]. Furthermore, the International Late Effects of Childhood Cancer Guideline Harmonization Group recently performed a comprehensive review of the evidence for cardiomyopathy surveillance in survivors of childhood cancer and concluded that echocardiographybased imaging is the preferred modality for surveillance among survivors treated with cardiotoxic therapies [39]. Additional studies are needed to evaluate if early detection of subclinical LV systolic dysfunction using 2DSTE can lead to improved cardiovascular outcomes among cancer survivors.

The findings of this study reinforce the importance of continued routine cardiac surveillance for adult survivors of childhood, adolescent, and young adult cancer many years beyond their initial treatment. Consistent with prior reports, LV systolic dysfunction was detected 15 years after successful treatment in this study, despite only modest doses of anthracycline (median $300 \mathrm{mg} / \mathrm{m}^{2}$ ) exposure. Lipshultz et al. also demonstrated that parameters of LV function progressively declined as many as 15 years after diagnosis among childhood cancer survivors treated with doxorubicin [11]. Given the high prevalence of subclinical LV systolic dysfunction in our study and the potential associated late cardiovascular consequences, continued periodic cardiac surveillance among adult cancer survivors should be an essential component of their long-term care.

This study has several limitations. The prognostic significance of abnormal GLS was not evaluated, and longterm follow-up is currently underway to determine whether 
abnormal GLS is predictive of subsequent LVEF decline or symptomatic heart failure in this population. Given the limitations of a retrospective cross-sectional study, we assessed LV function at one point in time and cannot comment on longitudinal changes that may have occurred. An analysis of serial echocardiograms is currently underway to investigate the natural history of GLS and LVEF impairment in this atrisk patient population. Although the study did not include a healthy control group, we used reference normative strain data that have been published in several healthy cohorts. Blood pressures were not measured at the time of the echocardiogram which can cause fluctuation in the GLS, but the majority of the patients were normotensive. Finally, our study pools together a heterogeneous study population with multiple cancer types from a single cancer referral center, which may limit the generalizability of the results.

LV systolic dysfunction is significantly more prevalent when assessed by GLS than by $2 \mathrm{D}$ LVEF among adult survivors of childhood, adolescent, and young adult cancer. 2DSTE may be useful for the long-term follow-up in this high risk population to identify patients with subclinical LV systolic dysfunction despite a normal LVEF. Mediastinal radiotherapy appears to be an independent risk factor for the development of LV systolic dysfunction in patients treated with anthracycline chemotherapy, and additional studies are needed to further explore the effect of possible confounders. Whether early identification of subclinical LV systolic dysfunction using 2DSTE will translate into longterm cardiovascular benefits warrants further investigation.

\section{Conflict of Interests}

The authors declare that there is no conflict of interests regarding the publication of this paper.

\section{Acknowledgments}

This work was supported in part through the National Cancer Institute (K05CA160724, PI: Kevin C. Oeffinger), the NIH/NCI Cancer Center Support Grant (P30 CA008748), and the Meg Berté Owen Foundation.

\section{References}

[1] N. N. A. Howlander, M. Krapcho, J. Garshell et al., Eds., SEER Cancer Statistics Review, 1975-2010, National Cancer Institute, Rockville, Md, USA, 2013, http://seer.cancer.gov/csr/ 1975_2010/.

[2] C. Parry, E. E. Kent, A. B. Mariotto, C. M. Alfano, and J. H. Rowland, "Cancer survivors: a booming population," Cancer Epidemiology Biomarkers and Prevention, vol. 20, no. 10, pp. 1996-2005, 2011.

[3] G. T. Armstrong, Q. Liu, Y. Yasui et al., "Late mortality among 5 -year survivors of childhood cancer: a summary from the childhood cancer survivor study," Journal of Clinical Oncology, vol. 27, no. 14, pp. 2328-2338, 2009.

[4] A. C. Mertens, Q. Liu, J. P. Neglia et al., "Cause-specific late mortality among 5-year survivors of childhood cancer: the childhood cancer survivor study," Journal of the National Cancer Institute, vol. 100, no. 19, pp. 1368-1379, 2008.

[5] R. C. Reulen, D. L. Winter, C. Frobisher et al., "Long-term causespecific mortality among survivors of childhood cancer," The Journal of the American Medical Association, vol. 304, no. 2, pp. 172-179, 2010.

[6] A. C. Mertens, Y. Yasui, J. P. Neglia et al., "Late mortality experience in five-year survivors of childhood and adolescent cancer: the Childhood Cancer Survivor study," Journal of Clinical Oncology, vol. 19, no. 13, pp. 3163-3172, 2001.

[7] K. C. Oeffinger, A. C. Mertens, C. A. Sklar et al., "Chronic health conditions in adult survivors of childhood cancer," The New England Journal of Medicine, vol. 355, no. 15, pp. 1572-1582, 2006.

[8] M. H. Chen, S. D. Colan, and L. Diller, "Cardiovascular disease: cause of morbidity and mortality in adult survivors of childhood cancers," Circulation Research, vol. 108, no. 5, pp. 619628, 2011.

[9] M. J. Adams, S. R. Lipsitz, S. D. Colan et al., "Cardiovascular status in long-term survivors of Hodgkin's disease treated with chest radiotherapy," Journal of Clinical Oncology, vol. 22, no. 15, pp. 3139-3148, 2004.

[10] S. E. Lipshultz, D. C. Landy, G. Lopez-Mitnik et al., "Cardiovascular status of childhood cancer survivors exposed and unexposed to cardiotoxic therapy," Journal of Clinical Oncology, vol. 30, no. 10, pp. 1050-1057, 2012.

[11] S. E. Lipshultz, S. R. Lipsitz, S. E. Sallan et al., "Chronic progressive cardiac dysfunction years after doxorubicin therapy for childhood acute lymphoblastic leukemia," Journal of Clinical Oncology, vol. 23, no. 12, pp. 2629-2636, 2005.

[12] V. I. Franco, J. M. Henkel, T. L. Miller, and S. E. Lipshultz, "Cardiovascular effects in childhood cancer survivors treated with anthracyclines," Cardiology Research and Practice, vol. 2011, Article ID 134679, 13 pages, 2011.

[13] C. Jaworski, J. A. Mariani, G. Wheeler, and D. M. Kaye, "Cardiac complications of thoracic irradiation," Journal of the American College of Cardiology, vol. 61, no. 23, pp. 2319-2328, 2013.

[14] G. T. Armstrong, M. Stovall, and L. L. Robison, "Longterm effects of radiation exposure among adult survivors of childhood cancer: results from the Childhood Cancer Survivor Study," Radiation Research, vol. 174, no. 6, pp. 840-850, 2010.

[15] Children's Oncology Group, Long-Term Follow-Up Guidelines for Survivors of Childhod, Adolescent and Young Adult Cancers, Version 3.0, Children's Oncology Group, 2008, http://www.survivorshipguidelines.org.

[16] P. F. Coccia, J. Altman, S. Bhatia et al., "Adolescent and young adult oncology. Clinical practice guidelines in oncology," Journal of the National Comprehensive Cancer Network, vol. 10, no. 9, pp. 1112-1150, 2012.

[17] P. Thavendiranathan, A. D. Grant, T. Negishi, J. C. Plana, Z. B. Popović, and T. H. Marwick, "Reproducibility of echocardiographic techniques for sequential assessment of left ventricular ejection fraction and volumes: application to patients undergoing cancer chemotherapy," Journal of the American College of Cardiology, vol. 61, no. 1, pp. 77-84, 2013.

[18] M. S. Ewer and D. J. Lenihan, "Left ventricular ejection fraction and cardiotoxicity: is our ear really to the ground?" Journal of Clinical Oncology, vol. 26, no. 8, pp. 1201-1203, 2008.

[19] B. W. Eidem, "Identification of anthracycline cardiotoxicity: left ventricular ejection fraction is not enough," Journal of the American Society of Echocardiography, vol. 21, no. 12, pp. 12901292, 2008. 
[20] Y.-F. Cheung, W.-J. Hong, G. C. F. Chan, S. J. Wong, and S.-Y. $\mathrm{Ha}$, "Left ventricular myocardial deformation and mechanical dyssynchrony in children with normal ventricular shortening fraction after anthracycline therapy," Heart, vol. 96, no. 14, pp. 1137-1141, 2010.

[21] J. T. Poterucha, S. Kutty, R. K. Lindquist, L. Li, and B. W. Eidem, "Changes in left ventricular longitudinal strain with anthracycline chemotherapy in adolescents precede subsequent decreased left ventricular ejection fraction," Journal of the American Society of Echocardiography, vol. 25, no. 7, pp. 733740, 2012.

[22] N. Fallah-Rad, J. R. Walker, A. Wassef et al., "The utility of cardiac biomarkers, tissue velocity and strain imaging, and cardiac magnetic resonance imaging in predicting early left ventricular dysfunction in patients with human epidermal growth factor receptor II-positive breast cancer treated with adjuvant trastuzumab therapy," Journal of the American College of Cardiology, vol. 57, no. 22, pp. 2263-2270, 2011.

[23] E. Ho, A. Brown, P. Barrett et al., "Subclinical anthracycline- and trastuzumab-induced cardiotoxicity in the long-term followup of asymptomatic breast cancer survivors: a speckle tracking echocardiographic study," Heart, vol. 96, no. 9, pp. 701-707, 2010.

[24] J. C. Plana, M. Galderisi, A. Barac et al., "Expert consensus for multimodality imaging evaluation of adult patients during and after cancer therapy: a report from the American Society of Echocardiography and the European Association of Cardiovascular Imaging," Journal of the American Society of Echocardiography, vol. 27, no. 9, pp. 911-939, 2014.

[25] R. M. Lang, M. Bierig, R. B. Devereux et al., "Recommendations for chamber quantification: a report from the American Society of Echocardiography's Guidelines and Standards Committee and the Chamber Quantification Writing Group, developed in conjunction with the European Association of Echocardiography, a branch of the European Society of Cardiology," Journal of the American Society of Echocardiography, vol. 18, no. 12, pp. 1440-1463, 2005.

[26] V. Mizariene, S. Bucyte, D. Zaliaduonyte-Peksiene, R. Jonkaitiene, J. Vaskelyte, and R. Jurkevicius, "Left ventricular mechanics in asymptomatic normotensive and hypertensive patients with aortic regurgitation," Journal of the American Society of Echocardiography, vol. 24, no. 4, pp. 385-391, 2011.

[27] N. Reckefuss, T. Butz, D. Horstkotte, and L. Faber, "Evaluation of longitudinal and radial left ventricular function by two-dimensional speckle-tracking echocardiography in a large cohort of normal probands," International Journal of Cardiovascular Imaging, vol. 27, no. 4, pp. 515-526, 2011.

[28] T. Yingchoncharoen, S. Agarwal, Z. B. Popović, and T. H. Marwick, "Normal ranges of left ventricular strain: a metaanalysis," Journal of the American Society of Echocardiography, vol. 26, no. 2, pp. 185-191, 2013.

[29] P. A. Heidenreich, S. L. Hancock, R. H. Vagelos, B. K. Lee, and I. Schnittger, "Diastolic dysfunction after mediastinal irradiation," American Heart Journal, vol. 150, no. 5, pp. 977-982, 2005.

[30] H. Sawaya, I. A. Sebag, J. C. Plana et al., "Assessment of echocardiography and biomarkers for the extended prediction of cardiotoxicity in patients treated with anthracyclines, taxanes, and trastuzumab," Circulation: Cardiovascular Imaging, vol. 5, no. 5, pp. 596-603, 2012.

[31] T. Stanton, R. Leano, and T. H. Marwick, "Prediction of all-cause mortality from global longitudinal speckle strain: comparison with ejection fraction and wall motion scoring," Circulation: Cardiovascular Imaging, vol. 2, no. 5, pp. 356-364, 2009.
[32] A. M. C. Mavinkurve-Groothuis, J. Groot-Loonen, K. A. Marcus et al., "Myocardial strain and strain rate in monitoring subclinical heart failure in asymptomatic long-term survivors of childhood cancer," Ultrasound in Medicine and Biology, vol. 36, no. 11, pp. 1783-1791, 2010.

[33] W. Yu, S.-N. Li, G. C. F. Chan, S.-Y. Ha, S. J. Wong, and Y.-F. Cheung, "Transmural strain and rotation gradient in survivors of childhood cancers," European Heart Journal Cardiovascular Imaging, vol. 14, no. 2, pp. 175-182, 2013.

[34] Y.-F. Cheung, S.-N. Li, G. C. F. Chan, S. J. Wong, and S.-Y. Ha, "Left ventricular twisting and untwisting motion in childhood cancer survivors," Echocardiography, vol. 28, no. 7, pp. 738-745, 2011.

[35] H.-K. Yu, W. Yu, D. K. L. Cheuk, S. J. Wong, G. C. F. Chan, and Y.-F. Cheung, "New three-dimensional speckle-tracking echocardiography identifies global impairment of left ventricular mechanics with a high sensitivity in childhood cancer survivors," Journal of the American Society of Echocardiography, vol. 26, no. 8, pp. 846-852, 2013.

[36] H.-R. Tsai, O. Gjesdal, T. Wethal et al., "Left ventricular function assessed by two-dimensional speckle tracking echocardiography in long-term survivors of Hodgkin's lymphoma treated by mediastinal radiotherapy with or without anthracycline therapy," The American Journal of Cardiology, vol. 107, no. 3, pp. 472-477, 2011.

[37] G. T. Armstrong, V. M. Joshi, K. K. Ness et al., "Comprehensive echocardiographic detection of treatment-related cardiac dysfunction in adult survivors of childhood cancer: results from the St. Jude Lifetime Cohort study," Journal of the American College of Cardiology, vol. 65, no. 23, pp. 2511-2522, 2015.

[38] P. Thavendiranathan, F. Poulin, K.-D. Lim, J. C. Plana, A. Woo, and T. H. Marwick, "Use of myocardial strain imaging by echocardiography for the early detection of cardiotoxicity in patients during and after cancer chemotherapy: a systematic review," Journal of the American College of Cardiology, vol. 63, no. 25, part A, pp. 2751-2768, 2014.

[39] S. H. Armenian, M. M. Hudson, R. L. Mulder et al., "Recommendations for cardiomyopathy surveillance for survivors of childhood cancer: a report from the International Late Effects of Childhood Cancer Guideline Harmonization Group," The Lancet Oncology, vol. 16, no. 3, pp. e123-e136, 2015. 


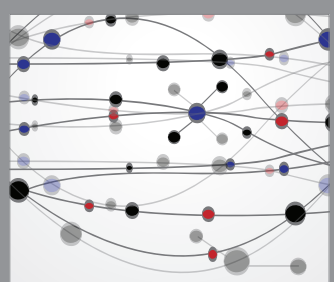

The Scientific World Journal
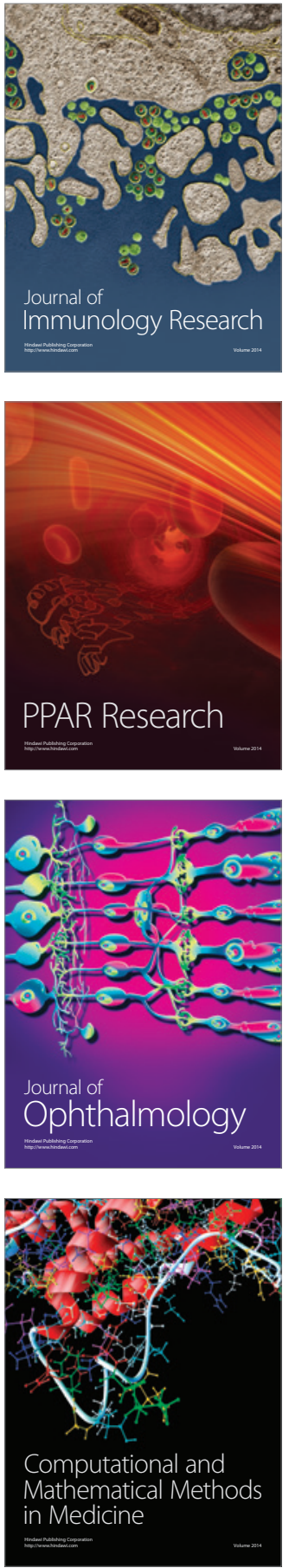

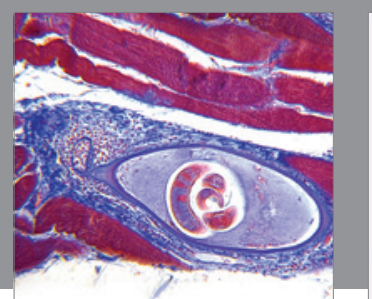

Gastroenterology Research and Practice

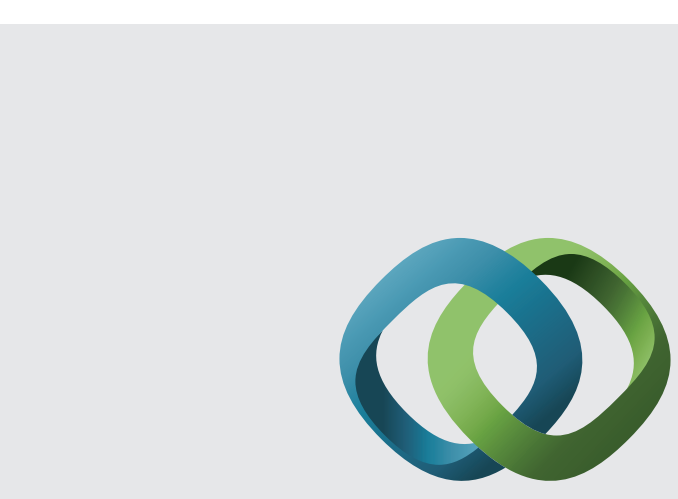

\section{Hindawi}

Submit your manuscripts at

http://www.hindawi.com
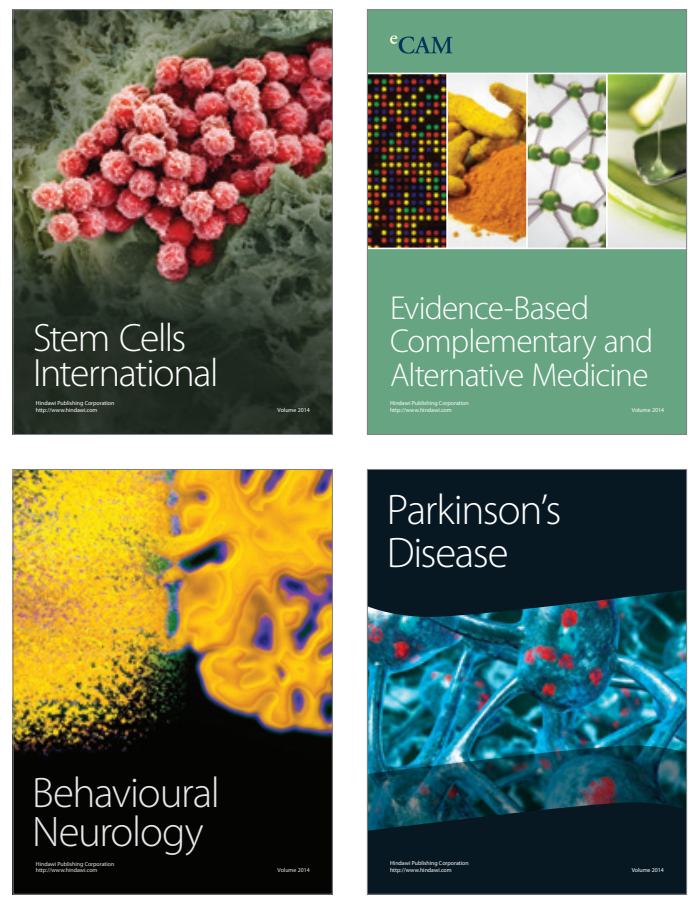
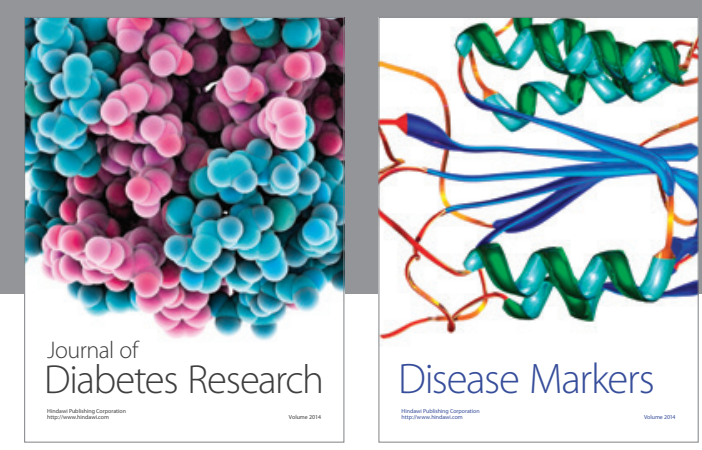

Disease Markers
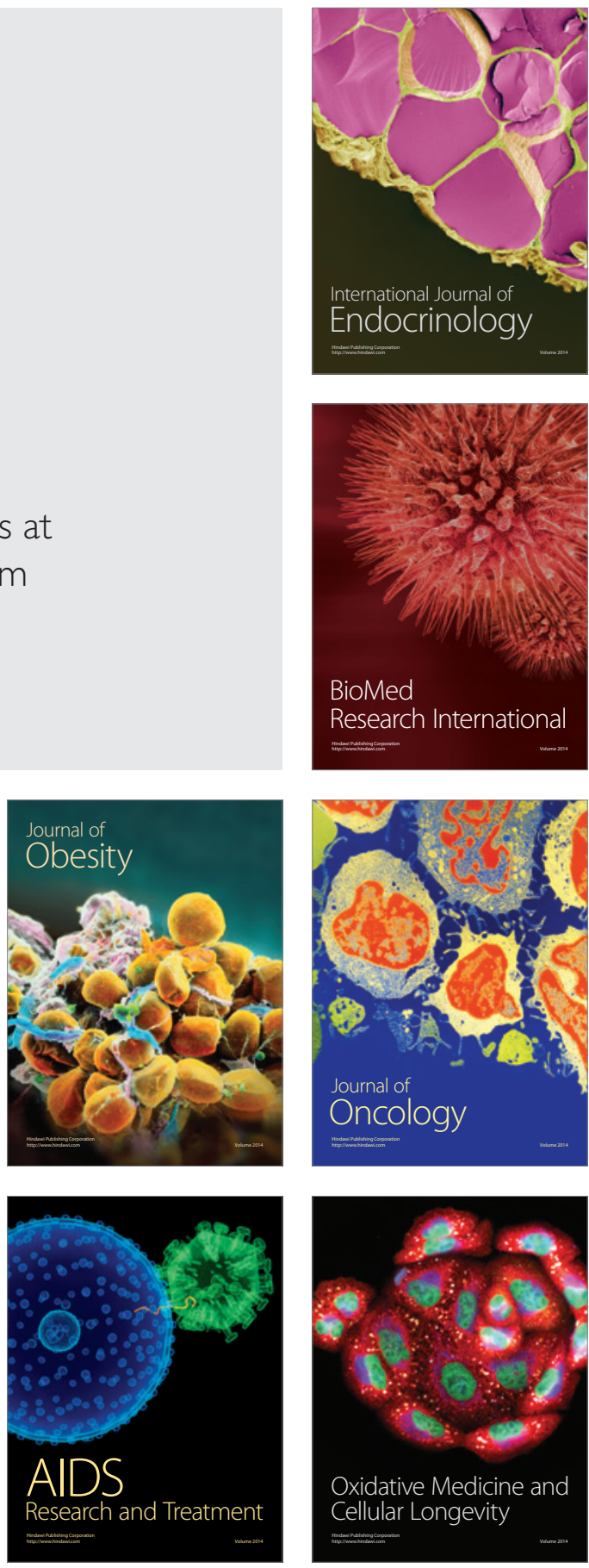